\title{
Declaração de Consenso da Seção de Psiquiatria Geriátrica da Associação Mundial de Psiquiatria sobre Ética e Capacidade em pessoas idosas com doença mental
}

World Psychiatric Association Section of Old Age Psychiatry Consensus Statement on Ethics and Capacity in older people with mental disorders

\author{
Cornelius Katona ${ }^{1}$, Edmond Chiuㄹ, Simon Adelman ${ }^{1}$, Stavros Baloyannis 3 , Vincent Camus 4 , Horácio Firmino ${ }^{5}$, \\ Dianne Gove 6 , Nori Graham 7 , Tesfamicael Ghebrehiwet ${ }^{8}$, Ilkin Icelli ${ }^{9}$, Ralf Ihl ${ }^{10}$, Aleksandra Milicevic Kalasic ${ }^{11}$, \\ Jerzy Leszek ${ }^{12}$, Scott Y. H. Kim ${ }^{13}$, Carlos Augusto de Mendonça Lima ${ }^{14}$, Carmelle Peisah ${ }^{15}$, Nicoleta Tataru ${ }^{16}$, \\ JAMES WARNER ${ }^{17}$
}

1 Departamento de Ciência da Saúde Mental, University College of London, Reino Unido.

2 Unidade Acadêmica de Psiquiatria da Terceira Idade, University of Melbourne, St. George's Hospital, Kew, Austrália.

3 Professor e chefe do Departamento de Neurologia, Aristotelian University, Tessalônica, Grécia.

${ }_{4}^{4}$ Professor. CHRU de Tours, Université Francois Rabelais de Tours, INSERM U930, França.

5 Professor. Clínica Psiquiátrica, Unidade de Gerontopsiquiatria, Hospitais da Universidade de Coimbra, Portugal.

6 Information Officer. Alzheimer Europe.

7 Vice-presidente honorária. Alzheimer Disease International.

8 Consultor. Nursing and Health Policy, International Council of Nurses, Genebra, Suiça.

${ }^{9}$ Departamento de Psiquiatria, Celal Bayar University, Faculty of Medicine, Manisa, Turkey.

10 Diretor da Unidade de Psiquiatria Geriátrica do Departamento de Psiquiatria, Alexian Hospital, Krefeld, University of Dusseldorf, Alemanha.

11 Instituto de Gerontologia, Beograd, Sérvia.

12 Departamento de Psiquiatria, Wroclaw University of Medicine, Wroclaw, Polônia

13 University of Michigan e American Association for Geriatric Psychiatry, Estados Unidos.

14 Diretor do Departamento de Psiquiatria e Saúde Mental, Centro Hospitalar do Alto Ave EPE, Guimarães, Portugal.

15 Professora-associada, University of New South Wales, Sydney, Austrália.

${ }_{16}$ Forensic Psychiatry Hospital, Stei, Romênia.

17 St. Charles Hospital and Imperial College, London, Inglaterra.

\section{Resumo}

Contexto: A Seção de Psiquiatria Geriátrica da Associação Mundial de Psiquiatria (AMP), desde 1997, vem desenvolvendo Declarações de Consenso relevantes para a prática da Psiquiatria Geriátrica. Desde 2006, a Seção vem trabalhando para desenvolver uma Declaração de Consenso sobre Ética e Capacidade em pessoas idosas com transtornos mentais. Método: Uma Conferência de Consenso foi realizada em Praga em setembro de 2008. Organizada pela Seção de Psiquiatria da Pessoa Idosa da AMP, ela contou com a participação do International Council of Nurses, Alzheimer Europe e Alzheimer Disease International. Os participantes foram reconhecidos pela sua perícia nesse domínio e vieram de 11 países. Incluíam psiquiatras, uma neurologista, um enfermeiro e representantes de cuidadores familiares. Resultados: Após dois dias de reuniões e debate, redigiu-se um rascunho da declaração que foi submetida para análise nas diversas organizações/associações que participaram da reunião. Após as sugestões finais recolhidas, um texto definitivo foi preparado em inglês e publicado. A presente versão em português é da responsabilidade de dois participantes lusófonos da reunião, que são também coautores da declaração de consenso final. Conclusões: Essa Declaração de Consenso oferece aos clínicos em saúde mental que cuidam de pessoas idosas com transtornos mentais, cuidadores, outros profissionais da saúde e o público em geral as definições e o debate sobre os princípios éticos que podem frequentemente ser complexos e desafiadores, apoiados em orientações práticas para satisfazer tais necessidades e padrões éticos e encorajar a boa prática clínica.

Katona C, et al. / Rev Psiq Clín. 2010;37(4):157-61

Palavras-chave: Ética, capacidade, discernimento, psiquiatria geriátrica.

\begin{abstract}
Background: The World Psychiatric Association (WPA) Section of Old Age Psychiatry, since 1997, has developed Consensus Statements relevant to the practice of Old Age Psychiatry. Since 2006 the Section has worked to develop a Consensus Statement on Ethics and Capacity in older people with mental disorders. Method: A Consensus Conference was realized in Prague, September 2008. The meeting was organized by the WPA Section of Old Age Psychiatry, with the participation of International Council of Nurses, Alzheimer Europe and Alzheimer Disease International. Participants who are recognised for their expertise in this area came from 11 countries and include psychiatrists, a neurologist, a nurse and family caregiving representatives. Results: After two days of debate a draft was prepared and submitted to the organizations/associations represented at the meeting. When their respective comments were collected, a final text was prepared and published originally in English. This Portuguese version was prepared by two members of the meeting who came from Portuguese spoken countries. Discussion: This Consensus Statement offers to mental health clinicians caring for older people with mental disorders, caregivers, other health professionals and the general public the setting out of and discourse in ethical principles which can often be complex and challenging, supported by practical guidance in meeting such ethical needs and standards, and to encouraged good clinical practice.
\end{abstract}

Katona C, et al. / Rev Psiq Clín. 2010;37(4):157-61

Keywords: Ethics, capacity, old age psychiatry. 


\section{Introdução}

Este documento de consenso surge no seguimento de uma série de Declarações de Consenso da Seção de Psiquiatria da Pessoa Idosa da Associação Mundial de Psiquiatria (AMP) assim como das atividades da Seção desde 2006 no domínio da Ética. Contribui igualmente para o trabalho que a AMP tem desenvolvido sobre ética em saúde mental, em particular a Declaração de Hawai//I' ${ }^{1}$ a Declaração de Madrid sobre Padrões Éticos para a Prática da Psiquiatria ${ }^{2}$ e a Declaração e Pontos de Vista da AMP acerca dos Direitos e Proteções Legais dos indivíduos portadores de Doença Mental $\mathrm{III}^{3}$. O Grupo do Consenso se reuniu em Praga, República Tcheca, em setembro de 2008 para desenvolver essa declaração. A reunião foi organizada pela Seção de Psiquiatria da Pessoa Idosa da AMP, com a participação do International Council of Nurses, Alzheimer Europe e Alzheimer Disease International. Os participantes foram reconhecidos pela sua perícia nesse domínio e vieram de 11 países. Incluíam psiquiatras, uma neurologista, um enfermeiro e representantes de cuidadores familiares.

O objetivo dessa Declaração de Consenso é o de fornecer um instrumento prático para auxiliar todos aqueles que estão envolvidos em questões éticas. Ele é dirigido aos profissionais que prestam cuidados de saúde e de assistência social, médicos em formação, organizações profissionais, pessoas idosas, suas famílias e cuidadores, organizações não governamentais e outros grupos de defesa críveis de interesses, aos gestores dos serviços de saúde e responsáveis pelas políticas de saúde assim como para o público em geral. A prestação de cuidados de alta qualidade às pessoas idosas com doença mental levanta problemas éticos complexos; por exemplo, orientar a tomada de decisões suscitadas pela transferência de cuidados independentes para a institucionalização de um idoso e pela distribuição dos escassos recursos dos sistemas de saúde. Os profissionais de saúde mental têm uma responsabilidade particular em garantir que os idosos com problemas de saúde mental sejam envolvidos, num grau máximo, nas decisões sobre o seu tratamento e cuidados assistenciais, em salvaguardar os direitos dos seus pacientes e em combater os preconceitos sobre o envelhecimento e o abuso das pessoas idosas. Os objetivos dessa Declaração são promover o debate a todos os níveis acerca dessas questões éticas, descrever os principais contextos em que a prestação de cuidados de elevada qualidade a idosos com doença mental levanta problemas éticos complexos, atualizar a prática e a formação dos profissionais e encorajar uma defesa efetiva dos interesses dos doentes.

Neste documento o termo "doença mental" refere-se tanto às demências como às outras perturbações psiquiátricas que afetam as pessoas idosas, tal como tem sido nas precedentes Declarações de Consenso emanadas da Seção ${ }^{4-7}$. Por convenção, definiu-se como idosa a pessoa que atingiu a idade da aposentadoria ${ }^{4}$ mas esse documento é igualmente relevante para indivíduos mais jovens com demência dado que, em alguns países, a responsabilidade pelo apoio a esses doentes é dos serviços que prestam apoio às pessoas idosas.

\section{Princípios éticos}

Os profissionais devem sempre atuar respeitando a dignidade e a individualidade da pessoa idosa com doença mental. O conteúdo desse documento é guiado por princípios éticos universais; estes raramente operam isoladamente. Apesar de muitas decisões éticas difíceis envolverem princípios contraditórios, é possível encontrar uma resolução razoável.

\section{Respeito pela autonomia}

Consiste na necessidade de respeitar o direito do indivíduo à autodeterminação e às decisões que toma em relação aos cuidados de saúde e de apoio social, desde que possua a capacidade para fazê-lo. O objetivo das intervenções em saúde mental para pessoas idosas é preservar e reforçar a sua autonomia pessoal. O respeito pela autonomia deve ser universal, embora o processo de decisão para avaliar a capacidade possa variar consoante o país.
Por exemplo, desde que tenham capacidade, os indivíduos devem poder escolher se querem ou não tomar fármacos para a depressão.

\section{Beneficência/não maleficência}

A beneficência consiste na necessidade de considerar os benefícios potenciais que podem resultar de uma intervenção comparando-os com os riscos potenciais. Os clínicos devem sempre agir de modo a procurar o benefício dos doentes. A não maleficência é a necessidade de não causar dano.

Por exemplo, o médico que prescreve um fármaco para a depressão deve estar convicto de que a medicação será eficaz e deve tomar medidas para minimizar qualquer efeito adverso.

\section{Justiça}

Por justiça compreende-se à necessidade (ao nível local e governamental) de garantir a distribuição equitativa de recursos e de tratar de todos os doentes de forma semelhante.

Por exemplo, todos os doentes com depressão devem ter igual acesso a tratamento independentemente da idade e circunstâncias.

\section{Veracidade}

Os médicos devem ser honestos com os doentes, tendo estes o direito de conhecer (ou de não conhecer) os diagnósticos e de ter acesso a uma informação correta quando solicitada.

Por exemplo, ao prescrever uma terapêutica inicial para depressão um médico deve informar o doente do diagnóstico, prognóstico e benefícios/riscos associados ao tratamento proposto.

\section{Direitos humanos para pessoas idosas}

Existem algumas normas orientadoras que definem os Princípios de Direitos Humanos para Pessoas Idosas ${ }^{8}$ e para indivíduos com doença mental9 ${ }^{9}$ As organizações de profissionais da saúde desenvolveram também códigos de ética para o exercício da atividade que integram princípios éticos e os direitos humanos. Até ao momento não temos conhecimento de que tenham sido publicadas normas específicas sobre Direitos Humanos para Pessoas Idosas com problemas de saúde mental. No entanto, essas normas são necessárias considerando a vulnerabilidade especial dessa população face ao preconceito da sociedade em relação ao envelhecimento, a estigmatização, a exclusão assim como a incapacidade e a dependência que a doença mental na terceira idade pode acarretar.

Os seguintes valores devem fundamentar essas normas:

- Independência. As pessoas idosas com doenças mentais têm o direito de contribuir de forma útil para a sociedade e tomar as suas próprias decisões em matérias que afetam a sua vida e a sua morte.

- Segurança e dignidade. As pessoas idosas com doenças mentais têm o direito de viver em segurança, com condições adequadas de alimentação e de habitação, livres de violência, abuso, negligência e exploração.

- Apoio e tratamento. As pessoas idosas com doenças mentais devem se beneficiar do cuidado e proteção de suas famílias e da comunidade e ter acesso a um sistema de saúde que proporcione a manutenção ou recuperação do seu maior nível de funcionamento e de bem-estar e previna ou retarde a deterioração.

\section{Preconceito em relação a idade, discriminação e estigmatização}

Esta Declaração afirma os princípios enunciados numa anterior Declaração de Consenso da OMS/AMP7.

As seguintes definições estão na base desses princípios. 
O estigma resulta de um processo por meio do qual certas pessoas e grupos de pessoas são levados a se sentirem envergonhados, excluídos e discriminados.

A discriminação se refere a toda distinção, exclusão ou preferência que tem como efeito a abolição ou diminuição do gozo equitativo de direitos.

O preconceito e a discriminação em relação à idade descrevem atitudes e práticas que causam desvantagens às pessoas idosas.

As pessoas idosas com doença mental podem experimentar o duplo prejuízo da estigmatização por terem uma doença mental e por serem idosos. Em alguns países, as mulheres idosas podem ter de enfrentar um estigma adicional em consequência do gênero. A pobreza pode conduzir a uma discriminação suplementar. A necessidade de reduzir o estigma e a discriminação é um imperativo ético imposto pelas suas consequências lesivas nomeadamente a perpetuação e agravamento da doença mental ${ }^{9}$. Nem a idade cronológica nem a demência (ou incapacidade mental) devem ser, por si próprias, motivo para suspender tratamentos benéficos para condições psiquiátricas ou físicas. A escassez geral de recursos não justifica a discriminação contra as pessoas idosas com doença mental.

\section{Confidencialidade}

Os indivíduos têm o direito de esperar que a informação a seu respeito seja tratada de forma confidencial. A confidencialidade deve ser protegida no caso de pessoas que não possuem capacidade de discernimento exceto se existirem receios justificados sobre o seu bem-estar ou sobre a segurança de outros. O grau de cada rompimento da confidencialidade deve ser proporcional segundo a necessidade. O grau de informação que se considera aceitável fornecer aos familiares varia de acordo com as diferentes culturas.

Por exemplo, se um doente com psicose pede ao médico que não forneça detalhes do diagnóstico ou do tratamento a outras pessoas, o clínico deve manter a confidencialidade exceto quando a segurança de outra pessoa possa estar em risco em resultado da psicose.

\section{Maus-tratos em idosos}

As pessoas idosas com problemas de saúde mental apresentam elevado risco de ser vítimas de maus-tratos e abuso. Existem diversas formas de maus-tratos e abuso contra as pessoas idosas nomeadamente psicológico, físico, sexual, financeiro e social, bem como negligência e abandono. $\mathrm{O}$ abuso dos idosos é frequentemente ocultado e pouco denunciado. É uma obrigação de todas as pessoas envolvidas nos cuidados para idosos vulneráveis de permanecerem atentos aos sinais de abuso e os cuidadores devem reconhecer que a sobrecarga ligada aos cuidados pode reduzir o limiar para perpetrar abusos.

$\mathrm{O}$ abuso não deve ser ignorado, quaisquer que sejam as circunstâncias atenuantes. $\mathrm{O}$ que pode ser considerado como não abusivo para um indivíduo saudável e com discernimento pode sê-lo no caso de um adulto idoso vulnerável. A provisão de um número adequado de profissionais, com formação apropriada, recebendo salário justo e o reconhecimento pelo trabalho feito, e uma definição dos planos de carreiras são elementos essenciais para reduzir o risco de abuso de idosos, assim como o aumento da consciencialização e da educação da população geral.

\section{Capacidade para tomar as decisões}

Quase todos os ordenamentos jurídicos possuem estratégias legais para proteger as pessoas com uma diminuição da capacidade de decisão. Apesar de terem sido propostas definições separadas para "capacidade" e "competência", na prática esses termos são usados como sinônimos para designar uma aptidão suficiente para tomar decisões informadas. Nem a idade nem o diagnóstico de doença mental são suficientes em si mesmo para determinar a ausência de capacidade.

A presunção da capacidade é um princípio legal importante, embora, em alguns sistemas jurídicos, a presença de transtornos mentais graves possa reverter essa presunção. É importante realçar que a capacidade é específica em relação (a) à decisão em causa (por exemplo, decisões acerca dos cuidados de saúde, testamentos escritos, gestão financeira) e (b) à sua complexidade (por exemplo, um indivíduo pode ter capacidade de consentir a administração de analgésicos para cefaleias, mas pode não ter capacidade de fornecer consentimento para ser submetido à eletroconvulsoterapia). A capacidade pode também alterar-se ao longo do tempo e de acordo com o contexto. Uma boa prática clínica exige uma abordagem flexível e de apoio, a fim de otimizar a capacidade.

A maioria dos profissionais de saúde e de apoio social que trabalham com idosos terá de realizar algum tipo de avaliação de capacidade e deve, por isso, receber formação apropriada. Poderá ser necessário obter o aconselhamento de peritos nos casos em que os profissionais sejam chamados a pronunciar-se formalmente sobre a existência de capacidade, de acordo com os costumes locais e preceitos legais.

As pessoas idosas que têm competência para tomar decisões têm o direito de recusar tratamentos que considerem que poderiam comprometer a sua qualidade de vida. Os indivíduos deverão receber a informação adequada e de forma apropriada acerca da decisão em causa. A informação deve incluir o estado do doente, os possíveis benefícios e riscos da intervenção proposta e as alternativas disponíveis e suas consequências potenciais. Os elementos-chave da capacidade de decisão incluem a compreensão, apreciação, julgamento, retenção e a aptidão de expressar preferência de forma consistente. $O$ fato de uma pessoa estar tomando uma decisão aparentemente imprudente ou irracional (por exemplo, agir contra conselho médico) não torna, em si mesmo, a pessoa incompetente.

A capacidade de gerir assuntos pessoais e financeiros é determinada pelo dispositivo jurídico local informado por meio de uma avaliação clínica e funcional global que inclui, mas não se restringe, a avaliação cognitiva.

\section{Tomadas de decisão por um substituto em assuntos pessoais, cuidados de saúde e financeiros}

Os procedimentos de designação de um substituto para tomada de decisões (STD) vão desde a transferência informal de fato da decisão para alguém nomeado pelo próprio doente quando ainda tem competência (incluindo o poder de representação legal) até os casos em que estes são designados por decisão judicial. Os termos "tutela/ curatela", "representação/defesa de interesses" e "proteção ou gestão financeira" têm diferentes significados consoante a jurisdição, mas habitualmente implicam a designação judicial de um STD relativas a assuntos pessoais, cuidados de saúde e aspectos financeiros. A Rede Europeia de Consenso para a Demência forneceu orientações úteis a esse respeito ${ }^{10}$. As recomendações-chave são apresentadas a seguir.

Deve ser permitido aos indivíduos com limitação de capacidade continuar a tomar as decisões em que mantêm a competência para o fazer por si próprios. Deve-se recorrer a um substituto para tomada de decisões apenas como último recurso, limitando-as às áreas sobre as quais o indivíduo não apresenta capacidade. Devem ser sempre consideradas outras abordagens menos restritivas em relação à gestão de assuntos pessoais e financeiros, tal como a nomeação de um representante legal (nota dos tradutores: por procuração).

A designação de um STD não deve implicar automaticamente a perda de outros poderes legais (por exemplo, votar, testar ou casar). Devem ser tomadas as medidas apropriadas para garantir e manter a discrição e o respeito pela privacidade e dignidade dos adultos com um STD. A pessoa incapacitada deve participar, tanto quanto possível, na escolha do seu substituto. No caso de substitutos designados judicialmente, a pessoa com incapacidade deve ser ouvida pelo juiz ou autoridade equivalente que ordena a nomeação de um substituto.

O STD deve levar em consideração as vontades da pessoa incapacitada. Isso deve incluir os desejos previamente manifestados (expressados em declarações antecipadas ou revelados por pessoas significativas). A pessoa deve ser continuamente informada e consultada acerca das decisões que são tomadas em seu nome, mesmo quando considerada mentalmente incapaz. 
As medidas e as ações feitas pelo STD devem ser implementadas no interesse da pessoa incapacitada e a necessidade da sua continuação deve ser revista regularmente. A gestão de bens deve ter como objetivo o benefício presente da pessoa sujeita ao STD e quaisquer conflitos de interesses devem ser resolvidos.

Devem existir mecanismos para recurso e revisão, bem como para denunciar alegados abusos dos STD. É recomendado que os STD nomeados judicialmente sejam reconhecidos como tal em outros países, exceto nos casos em que isso atente contra as leis de algum dos países. A aplicação dessas recomendações seria facilitada pela ratificação mais generalizada da Convenção de Haia sobre Proteção Internacional de Adultos ${ }^{11}$.

\section{Tomadas de decisão e relações afetivas}

De um lado as decisões das pessoas idosas com doença mental podem ser influenciadas pelas suas relações afetivas e, por outro, a doença mental pode afetar a tomada de decisões quanto ao estabelecimento ou ao fim de relacionamentos. $\mathrm{O}$ direito a estabelecer relacionamentos, incluindo de caráter sexual, constitui um direito humano fundamental acerca do qual tribunais, família e profissionais de saúde ou de assistência social não devem interferir, a menos que o exercício desse direito envolva abuso, negligência ou exploração. O objetivo primordial é promover a autonomia e garantir a segurança no seio da relação.

A decisão de iniciar uma relação afetiva é, obviamente, uma decisão pessoal que habitualmente não carece de um teste formal de avaliação de capacidade. No entanto, questões quanto a capacidade em consentir ou o risco de abuso podem às vezes surgir quando pessoas idosas com doença mental iniciam ou mantêm relações e os profissionais da saúde podem por vezes ser solicitados para avaliar ou intervir nessas situações. As considerações a serem levadas em conta no processo de decisão de intervenção ou de não intervenção numa possível relação abusiva envolvendo uma pessoa idosa com diminuição de capacidade englobam uma avaliação exaustiva do contexto relacional do indivíduo e da sua história de vida. Algumas questões específicas a considerar podem incluir:

- que tipo de relacionamento existe entre as duas pessoas? Existe um desequilíbrio de poder ou um elemento de coerção?

- existe uma discrepância significativa entre a capacidade cognitiva das suas pessoas?

- qual é o prazer (ou benefício) que a pessoa vulnerável experimenta no relacionamento? Eles manifestam intenção ou agrado de continuar a relação?

Os relacionamentos podem também constituir uma oportunidade para influenciar indevidamente nas tomadas de decisão de uma pessoa idosa. Os profissionais que trabalham com pessoas idosas com doenças mentais devem estar conscientes da possibilidade de influência de outras pessoas que procuram persuadir indivíduos mentalmente incapacitados para tomar decisões ou executar documentos legais em seu favor.

Para além de promover a segurança nos relacionamentos afetivos, essa declaração afirma a importância amplamente reconhecida do apoio, social nas vidas dos idosos com doença mental e o papel das organizações de apoio. Os cuidadores necessitam, eles próprios, de apoio e a integridade e o bem-estar do indivíduo que recebe cuidados dependem muitas vezes do bem-estar do cuidador. Podem surgir dificuldades especiais quando a saúde e a capacidade de decisão do cuidador (que é habitualmente o substituto na tomada de decisões) estão comprometidas.

\section{Aspectos sobre o fim da vida}

A demência é uma doença terminal. As pessoas com demência possuem necessidades relativas ao fim da vida semelhantes aos doentes com câncer terminal e devem ter acesso a serviços apropriados de cuidados paliativos. Pessoas com demência frequentemente recebem cuidados de fim de vida insuficientes. Os problemas cognitivos e de comunicação dificultam a prestação de cuidados paliativos adequados que devem, ainda assim, estar prontamente disponíveis. A dimensão espiritual faz parte integrante de cuidados de fim de vida de elevada qualidade. A depressão (que é potencialmente tratável) deve ser sempre considerada em idosos que procuram a eutanásia. O psiquiatra, cujos doentes podem estar gravemente incapacitados e sem competência para tomar uma decisão informada, devem ser particularmente cautelosos relativamente a ações que podem conduzir à morte daqueles que não podem proteger-se em razão de suas deficiências ${ }^{2}$. As declarações antecipadas (ou desejos anteriormente manifestados de forma clara) relativamente à suspensão de tratamentos devem ser, no entanto, respeitadas. As atitudes e a legislação sobre suicídio assistido/eutanásia variam amplamente e a prática deve adaptar-se às leis existentes e aos códigos éticos de conduta. Deve-se ter presente que (em contraste com preconceitos da população) a qualidade de vida pode ser boa, apesar da deterioração progressiva da função cognitiva.

\section{Participação em pesquisas}

A evidência científica para muitas terapêuticas psiquiátricas é inexistente para pessoas idosas e é necessário aprofundar a pesquisa. É necessário que essa pesquisa envolva pessoas idosas com doenças mentais. As pessoas idosas com doenças mentais devem ter o direito (e a oportunidade) de fazer parte de projetos de pesquisa, mesmo se não tenham capacidade a consentir. Essa capacidade pode melhorar por meio do fornecimento de informação sob forma que seja facilmente compreensível. Todavia, devem ser tomadas medidas de salvaguarda para garantir que os participantes que não tenham capacidade para dar o seu consentimento informado sejam protegidos. Os substitutos para a tomada de decisões relativas à saúde devem possuir a autoridade para dar o consentimento informado por procuração, exceto nos casos em que a legislação específica em vigor determine o contrário. As pessoas que estão preparando decisões/declarações antecipadas devem ser encorajadas a incluir uma declaração sobre os seus desejos relativamente à participação em projetos de pesquisa. Os profissionais de saúde mental envolvidos em estudos ou aconselhamento genéticos devem ter em mente que a informação genética tem implicações que ultrapassam o participante individual e que podem ter efeitos negativos e disruptivos na família e comunidade².

\section{Conclusão}

A consideração dos aspectos éticos é um componente essencial da boa prática clínica. Os aspectos éticos são frequentemente complexos e algumas vezes conflitantes. Este documento ajudará os clínicos a compreenderem os princípios e sintetiza as nuances éticas que eles enfrentam todas as vezes em que encontram seus pacientes.

\section{Conflito de interesses}

A Conferência de Consenso recebeu um fundo educacional irrestrito da Servier Pharmaceuticals e apoio financeiro da Unidade Acadêmica para Psiquiatria Geriátrica da Universidade de Melbourne.

\section{Participantes da conferência de consenso}

Aleksandra Milicevic Kalasic

Instituto de Gerontologia

Beograd, Serbia.

Carlos Augusto de Mendonça Lima

Departamento de Psiquiatria e Saúde Mental

Centro Hospitalar do Alto Ave

Guimarães, Portugal.

Carmelle Peisah

Professora-associada

University of New South Wales

Sydney, Austrália. 
Cornelius Katona

Department of Mental Health Science

University College of London.

Londres, Reino Unido.

\section{Dianne Gove}

Information Officer

Alzheimer Europe.

Edmond Chiu

Academic Unit for Psychiatry of old Age

University of Melbourne

Melbourne, Austrália.

Horácio Firmino

Unidade de Gerontopsiquiatria, Clínica Psiquiátrica

Hospitais Universitários de Coimbra

Coimbra, Portugal.

Ilkin Icelli

Departamento de Psiquiatria

University of Celal Bayar

Manisa, Turquia.

James Warner

St. Charles Hospital and Imperial College

Londres, Reino Unido.

Jerzy Leszek

Departamento de Psiquiatria

Wroclaw University of Medicine,

Wroclaw, Polônia.

Nicoleta Tataru

Forensic Psychiatry Hospital, Stei,

Romênia.

Nori Graham

Honorary vice president, Alzheimer

Disease International.

Ralf Ihl

Department of Psychiatry and Head

of the Geriatric Psychiatry Unit,

Alexian Hospital, Krefeld, University

of Dusseldorf, Germany.

Scott Y. H. Kim

University of Michigan and

American Association for Geriatric

Psychiatry, Estados Unidos.

Simon Adelman

Department of Mental Health

Sciences, University College of

London, Reino Unido.

Stavros Baloyannis

Professor e chefe do Departamento de Neurologia

Aristotelian University

Tessalônica, Grécia.
Tesfamicael Ghebrehiwet

Consultort.

Nursing and Health Policy

International Council of Nurses

Genebra, Suíça.

Vincent Camus

CHRU de Tours

Université François Rabelais

INSERM U930

Tours, França.

Tradução para a língua portuguesa autorizada pelo editor do documento original ${ }^{12}$ :

Carlos Augusto de Mendonça Lima e Horácio Firmino.

\section{Referências}

1. World Psychiatric Association. Declaration of Hawaii/II. Approved by the General Assembly of the World Psychiatric Association in Vienna, Austria, on 10th July 1983. Disponível em: http://www.wpanet.org/detail. php?section_id=5\&content_id=27. Acesso em: 30 jun 2010.

2. World Psychiatric Association. Madrid Declaration on Ethical Standards for Psychiatric Practice. Approved by the General Assembly of the World Psychiatric Association in Madrid, Spain, on August 25, 1996, and enhanced by the WPA General Assemblies in Hamburg, Germany on August 8, 1999, in Yokohama, Japan, on August 26, 2002, and in Cairo, Egypt, on September 12, 2005. Disponível em: 6.http://www.wpanet.org/detail. php? section_id=5\&content_id=48. Acesso em: 30 jun 2010.

3. World Psychiatric Association. WPA Statement and Viewpoints on the Rights and Legal Safeguards of the Mentally Ill. Adopted by the WPA General Assembly in Athens, 17th October, 1989. Disponível em: http:// www.wpanet.org/detail.php?section_id=5\&content_id=29. Acesso em: 30 jun 2010.

4. World Health Organization/World Psychiatric Association. Psychiatry of the Elderly: a Consensus Statement. WHO, Geneva, 1996. WHO/ $\mathrm{MNH} / \mathrm{MND} / 96.7$.

5. World Health Organization/World Psychiatric Association. Organization of Care in Psychiatry of the Elderly: a Technical Consensus Statement. WHO, Geneva, 1997. WHO/MSA/MNH/97.3.

6. World Health Organization/World Psychiatric Association. Education in Psychiatry of the Elderly: A Technical Consensus Statement. WHO, Geneva, 1998. WHO/MNH/MND/98.4.

7. World Health Organization/World Psychiatric Association. Reducing Stigma and Discrimination Against Older People With Mental Disorders: a Technical Consensus Statement. WHO, Geneva, 2002. WHO/ $\mathrm{MSD} / \mathrm{MBD} / 02.3$.

8. United Nations. United Nations Principles for Older Persons. General Assembly resolution 46/91 of 16 December 1991. Disponível em: http://www2. ohchr.org/english/law/pdf/olderpersons.pdf. Acesso em: 30 jun 2010.

9. World Health Organization. WHO Resource Book on Mental Health, Human Rights and Legislation. WHO, Geneva, 2005.

10. European Dementia Consensus Network Consensus Statements. Disponível em: http://edcon-dementia.net/en/consensusoverview.php. Acesso em: 30 jun 2010.

11. Convention on the International Protection of Adults. Concluded 13 January 2000. Entered into force 1 January 2009. Disponível em: http:// www.hcch.net/index_en.php?act $=$ conventions.text\&cid=71. Acesso em: 30 jun 2010.

12. Katona C, Chiu E, Adelman S, Baloyannis S, Camus V, Firmino H, et al. World psychiatric association section of old age psychiatry consensus statement on ethics and capacity in older people with mental disorders. Int J Geriatr Psychiatry. 2009;24(12):1319-24. 